\section{NETCON: A general-purpose program for creation of a minimal length circuit matrix from sociometric data}

\author{
PERCY B. BROWN \\ School of Business Administration \\ University of California, Berkeley, California 94720
}

Sociometric analysis has been used in a variety of research settings to concisely represent and systematically explore patterns of relations among members of social collectivities. Such analysis typically seeks to better understand individuals' positions in social structures or to identify subsets of members that may exist within a collectivity. Researchers frequently obtain sociometric data directly from collectivity members through interviews or self-reports and, to a lesser extent, through observation of social interaction and analysis of archival information. As the size and complexity of collectivities increase, reliance upon self-reports by collectivity members becomes the only practical method of obtaining sociometric data.

Where sociometric data are acquired through selfreporting techniques, each respondent may be asked to indicate either the existence or the extensiveness of a criterion relation between himself and each of the other collectivity members. When a respondent is requested to indicate whether or not a criterion relation exists with other individuals in the collectivity, a binary sociomatrix results. However, when collectivity members are asked to rank or rate each other, a multivalued sociomatrix is produced. The cells of such a multivalued sociomatrix represent levels of relative choice intensity that exists between collectivity members, and such cells are often interpreted as perceived social distances between individuals.

Except for smaller and more simply structured collectivities, sociometric data obtained through selfreports represent respondents' localized views of proximities among collectivity members. Because the pattern of social relations is viewed from a local rather than a general perspective, and because of psychometric considerations involved in measuring the perceived strength of a relation between two collectivity members, the ability to infer directly social distances from raw sociometric data is greatly impaired. Brown (Note 1) argues that social networks that reflect the extensiveness of relations among collectivity members and are viewed from a general perspective will facilitate derivation of subcollectivities through application of multivariate analyses of interdependence. A series of graph-theoretic transformations, which may be applied to either binary or multivalued sociometric data to obtain the desired general perspective of relational patterns among collectivity members, is then described. These transformations are incorporated in the network conversion program (NETCON), which converts an original sociomatrix into a standardized matrix of minimal length circuits. The matrix of minimal length circuits that connect each pair of collectivity members provides the desired generalized perspective of relational patterns and may serve as input to multivariate analyses of interdependence.

Description. Given a social collectivity that contains $\mathrm{N}$ members, let the pattern of relationships among collectivity members be represented as a directed graph, $G$. Let $C(G)$ and $X(G)$ represent the sets of vertices and arcs of $G$, respectively, such that $\mathrm{C}(\mathrm{G})=\{1,2, \ldots, \mathrm{i}, \ldots, \mathrm{N}\}$, where $\mathrm{i}$ is the ith member of the collectivity, and $x_{i j} \varepsilon X(G)$, where $\mathrm{x}_{\mathrm{ij}}$ indicates a measure of sociometric choice given by collectivity member $i$ to collectivity member $j$. Note that the set of arcs $X(G)$ is identical to the sociomatrix $\mathbf{X}$ and that $x_{i j}$ is a cell in $\mathbf{X}$. Generally, $p \leqslant x_{i j}$ $\leqslant q$, where $p$ and $q$ define the range of responses of a rating scale. Typically, $\mathrm{p}, \mathrm{x}_{\mathrm{ij}}$, and $\mathrm{q}$ are integers. When collectivity members are required to indicate only whether or not the criterion relation exists, $0 \leqslant x_{i j} \leqslant 1$; when collectivity members are required to perform a strict rank ordering of collectivity members, $0 \leqslant x_{i j} \leqslant N-1$; and $x_{i j}=0$ when $\mathrm{i}=\mathrm{j}$. Although $p$ and $q$ may acquire any integer values, where $p<q$, NETCON requires that $p=0$. This requirement ensures that the concept of social proximity between any two collectivity members may be approximated by a sequence of positive integers. Therefore, if $x_{i j}=1$ and $x_{i k}=q$, then $j$ is perceived to be in closer proximity to $i$ than is $k$. NETCON assumes that necessary linear scale translations or transformations have been performed upon $\mathbf{X}$ to ensure that the requirement $0 \leqslant x_{i j} \leqslant q$ is met before $X$ is subjected to NETCON transformations.

The first NETCON transformation ensures comparability of choice intensity distributions by creating a modified sociomatrix $\mathbf{X}^{\prime}$ that contains standardized equal-interval choice distributions for each original row vector $x_{i}$. . Standardization of expressed choice intensities across collectivity members is accomplished through use of the scale conversion equation,

$$
\mathrm{x}_{\mathrm{ij}}^{\prime}=\left(\sigma_{\mathrm{i}}^{\prime} / \sigma_{\mathrm{i}}\right) \mathrm{x}_{\mathrm{ij}}-\left[\mu_{\mathrm{i}}\left(\sigma^{\prime} / \sigma\right)-\mu_{\mathrm{i}}\right]
$$


where $x_{i j}^{\prime}$ is a rescaled or converted choice value corresponding to $x_{i j} ; x_{i j}$ is an observed choice value contained in the jth cell of the ith row vector of the original sociomatrix $\mathbf{X} ; \mu_{\mathrm{i}}$ and $\mu_{\mathrm{i}}^{\prime}$ are the means of $x_{i j}$ and $x_{i j}^{\prime}$, for cells in row vectors $x_{i}$. and $x_{i}^{\prime}$, respectively; and $\sigma_{i}$ and $o_{i}^{\prime}$ are the standard deviations of $x_{i j}$ and $x_{i j}^{\prime}$ for cells in row vectors $x_{i}$. and $x_{i}^{\prime}$, respectively. Note that the scale conversion equation is applied only to cell values for which $x_{i j} \neq 0$, so that for each $\mathbf{x}_{\mathrm{i}}$.

$$
\mu_{i}=\frac{1}{n_{i}} \cdot \sum_{j=1}^{n_{i}} x_{i j}
$$

and

$$
\sigma_{i}=\left[\frac{1}{n_{i}} \cdot \sum_{j=1}^{n_{i}}\left(x_{i j}-\mu_{i}\right)^{2}\right]^{1 / 2},
$$

where $n_{i}$ is the number of cells in row vector $x_{i}$, , with $\mathrm{x}_{\mathrm{ij}}>0$. The values $\mu_{1}^{\prime}$ and $\sigma_{\mathrm{i}}^{\prime}$, which are common to all row vector distributions of nonzero cell values, may be provided by the user, or default values contained in the NETCON program may be employed.

NETCON provides for a maximally connected network $G\left(X^{\prime}\right)$ by creating artificial arcs of value $q N$ between points that are not initially connected. Therefore, when $\mathrm{i} \neq \mathrm{j}$ and $\mu_{\mathrm{i}}$ and $\sigma_{\mathrm{i}}$ have been computed over $n_{i}$ values, $q N$ is substituted for $x_{i j}$ in the scale conversion equation to obtain standardized maximal length paths in $x_{i j}^{\prime}$, where $x_{i j}=0$. When $\mathbf{X}$ contains only binary data (i.e., $q=1$ ), the scale conversion equation is not used and $\mathbf{X}^{\prime}$ is created by directly substituting $\mathrm{x}_{\mathrm{ij}}=\mathrm{N}$ for $\mathrm{x}_{\mathrm{ij}}=0$, where $\mathrm{i} \neq \mathbf{j}$.

The second NETCON transformation applies an algorithm described by Flament $(1963$, p. 60$)$ to $X^{\prime}$ in order to derive paths of minimal length from individual $i$ to individual $j$ for each pair of collectivity members. The resulting matrix of minimal length paths may be represented as $\Lambda$, where $\lambda_{\mathrm{ij}} \varepsilon \Lambda$. The creation of minimal length paths provides the desired general perspective on the pattern of relations among collectivity members.

The third NETCON transformation addresses difficulties created when $\lambda_{\mathrm{ij}} \neq \lambda_{\mathrm{ji}}$ (i.e., reciprocal paths between collectivity members $i$ and $j$ are not of equal length). This problem is resolved by considering the length of the closed path (i.e., citcuit) that links individual $i$ to $j$ and individual $j$ to $i$. A circuit of minimal length may be represented as

$$
\delta_{\mathrm{ij}}=\lambda_{\mathrm{ij}}+\lambda_{\mathrm{ji}}
$$

and the matrix of minimal length circuits for all pairs of collectivity members may be expressed as

$$
\Delta=\Lambda+\Lambda^{\mathrm{T}}
$$

where $\delta_{i j} \varepsilon \Delta$ and $\Lambda^{\mathrm{T}}$ is the transposition of $\Lambda$.

The final NETCON transformation applies T-scaling (Guilford \& Fruchter, 1973, p. 468) to the circuit matrix $\Delta$ to create standardized and normally distributed cell values in each column vector $\delta . \mathrm{j}$. The resulting modified circuit matrix is represented as $\Delta^{\prime}$. NETCON accomplishes T-scale transformations through use of the scientific subroutine package (International Business Machines, 1968) program NDTRI. The original distribution of cell values in each column vector of $\Delta$ is normalized by equating intervals, and thus proportionate corresponding areas, of the original distribution in $\delta_{. j}$ and a standard normal distribution.

Optional features of NETCON include: the capability of selecting any one of the four transformation steps, or combinations of two or three transformation steps, for application to an original sociomatrix; deletion of null column vectors $x_{\cdot j}$ and associated row vectors $x_{i}$. from the original sociomatrix prior to application of transformations; selective suppression of printed output; and storage of summarized output in machine-retrievable form.

Input. Program input consists of control specifications and data. The user may specify program options, title information, and a read format for row vectors of the sociomatrix, or he may rely upon default options contained within the program. The sociomatrix must be preceded by a header card, or card image, upon which is specified the number of row vectors in the sociomatrix and the maximum value that may be assumed by any null cell. Successive row vectors of the sociomatrix are then read as unit records. The first entry of each unit record is a label that uniquely identifies each row vector. The row vector label is followed by row vector cell values.

Output. Complete output includes a listing of input control information for verification of program options selected by the user, title information, and five matrices that result from the application of transformations. Output matrices include: (1) scaletranslated row vectors, (2) minimal length paths, (3) the number of intermediaries in each minimal length path from individual $i$ to individual $j$, (4) minimal length circuits, and (5) standardized normally distributed column vectors of minimal length circuits. A descriptive heading is generated for each output matrix, and each row and column vector is labeled. Each matrix may be stored in an output disk data file.

Limitations. The present version of NETCON is capable of processing an $\mathbf{N}$ by $\mathbf{N}$ sociomatrix in which ce" nay assume integer values that range 
between zero and eight. The size of the sociomatrix is limited only by restrictions on core availability at the user's computer facility.

Language and Computer. This program was developed at the University of California, San Francisco, on an IBM 370/148 with Virtual Machine Facility (VM)/370 Release 5. NETCON source code is written in FORTRAN IV, and object code is obtained from the FORTRAN G compiler. Although the program is not interactive, the IBM conversation monitor system (CMS) has been used for program editing, compilation, and execution.

Core and Time Requirements. The main program and associated subprograms require $21 \mathrm{~K}$ bytes of core. Necessary work space is approximated by $4\left(3 N^{2}+5 N\right)$, where $N$ is the size of the collectivity over which the sociomatrix is formed. Therefore, when $\mathrm{N}=25$, approximately $8 \mathrm{~K}$ bytes of core are needed for work space and maximum core required is about $30 \mathrm{~K}$ bytes. Work space may be adjusted according to the user's needs. NETCON requires $40 \mathrm{sec}$ for compilation under CMS. Where $\mathrm{N}=25$, approximately $10 \mathrm{sec}$ are required for program execution under CMS.

Availability. The NETCON user's guide, a current version of the program source code, and a test problem are available at no cost from Percy B. Brown, School of Business Administration, 350 Barrows
Hall, University of California, Berkeley, California 94720 .

\section{REFERENCE NOTE}

1. Brown, P. B. Decomposition of sociometric structure through canonical factor analysis of network circuits. Unpublished manuscript, University of California, Berkeley, 1979.

\section{REFERENCES}

Brown, P. B. BEMSD: A general-purpose program for estimating missing data in a sociomatrix. Behavior Research Methods \& Instrumentation, 1979, 11, 465-466.

Flament, C. Applications of graph theory to group structure. Englewood Cliffs, N.J: Prentice-Hall, 1963.

Guilford, J. P., \& Fruchter, B. Fundamental statistics of psychology and education. New York: McGraw-Hill, 1973.

International Business Machines. System 1360 scientific subroutine package (360A-CM-03X) version III programmer's manual (4th ed., H20-0205-3). White Plains, N.Y: IBM Technical Publication Department, 1968.

\section{NOTE}

1. Both NETCON and BEMSD (see Brown, 1979) result from continuing research on use of sociometric techniques in the analysis of structured networks in formal organizations. BEMSD is concerned with treatment of missing sociometric data. NETCON may use standardized sociometric data to identify minimal-length paths in a social network.

(Received for publication September 7, 1979; revision accepted October 17, 1979.) 